\title{
Immunolocalization of Vimentin in Macrophage-derived Giant Cells - Method for Obtaining Larger Giant Cells-
}

\author{
Shizuya Saika*, Shunsaku Ohmi*, Ryuichi Kanagawa*, Sai-ichi Tanaka*, \\ Yoshitaka Ohnishi, Akio Yamanaka** and Jun-ichi Hiraoka*** \\ *Department of Ophthalmology, and ${ }^{* * *}$ Department of Anatomy, Wakayama Medical College, 7-Bancho 27, \\ Wakayama, 640 and ${ }^{* *}$ Department of Ophthalmology, Kobe Kaisei Hospital, 3-11-15, Shinohara-Kitamachi, \\ Nada-Ku, Kobe, 675
}

Received for publication December 27, 1994 and in revised form March 3, 1995

\begin{abstract}
We studied the ultrastructure of giant cells derived from macrophages that are found on the surface of implanted polymethylmethacrylate intraocular lenses (IOLs) in rabbits and the immunolocalization of vimentin in these cells in order to study the rearrangement of the intermediate filaments in multinucleated cells. In adult Japanese albino rabbits, the crystalline lens was extracted and an $\mathrm{IOL}$ was inserted into the residual lens capsule. IOLs were removed after 7 days, and the specimens were processed for ultrastructural observation or for immunostaining for vimentin. Macrophages and multinucleated cell were detected on the
\end{abstract}

surfaces of IOLs by both light and transmission electron microscopy. Nuclei of the large multinucleated cells showed a circular distribution with the center of the cytoplasm occupied by cytoplasmic vacuoles. Vimentin intermediate filaments were observed in the cytoplasm of both macrophages and multinucleated cells. Vimentin in giant cells showed a radial distribution. Results indicate that vimentin intermediate filaments become reorganized after the fusion of macrophages. Experimental implantation of an $10 L$ into the animal eye is a useful method for obtaining larger giant cells derived from macrophages.

Key words: Macrophage, Giant cell, Vimentin, Intraocular lens, Rabbit

\section{Introduction}

After a cataractous lens is extracted from the eye, an artificial intraocular lens composed of polymethylmethacrylate (PMMA) is implanted to correct the refractive error caused by removal of the crystslline lens. Although the PMMA intraocular lens (IOL) induces a foreign body reaction, the rejecton reaction is mild and incomplete, and the IOL remains clear for a long period, resulting in a favorable clinical outcome.

Macrophages and macrophage-derived foreign body giant cells are found on the surface of explanted PMMA IOLs in animals and humans as the result of a foreign body reaction $[6-11,22,27]$. Similar multinucleated cells are formed on the surface of foreign bodies implanted in the peritoneal cavity of animals [1, 22-25]. However, the size of these multinucleated cells is quite different in the case of IOLs vs. that on foreign bodies implanted sub-

Correspondence to: Shizuya Saika, M.D., Department of Ophthalmology, Wakayama Medical College, 7-Bancho 27, Wakayama, 640, Japan. cutaneously or in the peritoneal cavity of animals. The number of the nuclei in multinucleated cells formed on the surface an IOL sometimes exceeds 500 , or 1000 , much greater than the number of foreign-body giant cells detected in the granulation tissue formed around foreign bodies implanted subcutaneously or in the peritoneal cavity.

Cytoskeletons are constructed of three types of intracytoplasmic fibers: microfilaments; intermediate filaments; and microtubules. Intermediate filaments are composed of cytokeratin in epithelial cells and of vimentin in mesenchymal cells including fibroblasts and macrophages. Actin and tubulin have been immunolocalized in macrophages and multinucleated giant cells in granulation tissue [3, 12-14, 21]. Intermediate filaments play an important role in the cytoplasm including the positioning of intracytoplasmic organellae and the support of the desmosomal structure. The reorganization of vimentin intermediate filaments in multinucleated cells has not been fully investigated [2], but it appears that intermediate filaments may be reconstructed after the fusion of many macrophages. 
We investigated (1) the presence of macrophages and foreign body giant cells on the surface of PMMA IOLs implanted experimentally in rabbit eyes after extraction of the crystalline lens, and (2) immunolocalization of vimentin in these cells by light microscopy after removal of the IOLs. The ultrastructure of giant cells was studied by transmission electron microscopy.

\section{Materials and Methods}

\section{Implantation of PMMA IOLs into rabbit eye}

Male or female Japanese albino rabbits, $2.0-2.5 \mathrm{~kg}$, $(n=7)$ were used. After induction of anesthesia with sodium pentobarbital, I.V., crystalline lens were removed bilaterally by the extracapsular method and a conventional PMMA IOL was inserted into the remaining lens capsule of each eye. Sodium hyaluronate $(1.0 \%)$ was injected into each eye to facilitate implantation of the IOLs. Seven days after the implantation, the implants were removed and either stained with hematoxylin-eosin (HE) for light microscopy $(n=4)$ or observed transmission electron microscopy $(n=4)$, or processed for immunohistochemistry to detect vimentin $(n=6)$.

\section{Morphological observation and vimentin immunohisto- chemistry \\ Explanted IOLs were fixed in $10 \%$ formalin and stain-} ed with $\mathrm{HE}$, or processed immunohistochemistry for vimentin. The fixed specimens were treated with mouse monoclonal anti-vimentin antibody $(\times 100$ in phosphate- buffered saline (PBS), Dakoppats, Denmark) after inactivation of endogenous peroxidase with methanol containing $0.1 \%$ hydrogen peroxide. After being washed in PBS, the IOLs were allowed to react with peroxidase-conjugated anti-mouse IgG antibody ( $\times 100$ in PBS, Cappel, OrganonTeknika, West Chester, PA, USA). After the IOLs were again washed in PBS, the color in the antigen-antibody complex was developed with $0.05 \%$ diaminobenzidine containing $0.06 \%$ hydrogen peroxide. Control staining was performed by ommision of the primary antibody. Specimens stained with HE or with anti-vimentin antibody were embedded in canada balsam and observed under light microscopy.

\section{Transmission electron microscopy}

Cells adhering to the surface of IOLs explanted from rabbit eyes were removed by trypsinization., and fixed in $1.0 \%$ osmium tetroxide in $0.1 \mathrm{M}$ phosphate buffer, $\mathrm{pH}$ 7.4. They were then collected by centrifugation at $2000 \mathrm{rpm}$ for $5 \mathrm{~min}$ and embedded in $2.0 \%$ agar. The agar block containing the cells was dehydrated through a graded seried of acetone solution and embedded in Epon 812 mixture. Ultrathin sections were cut, stained with uranyl acetate and lead citrate, and observed under transmission electron microscopy (JEOL SX-100).

\section{Results}

\section{$H E$ staining and immunohistochemistry for vimentin} Mononuclear macrophages (Fig. 1) and multinuclear

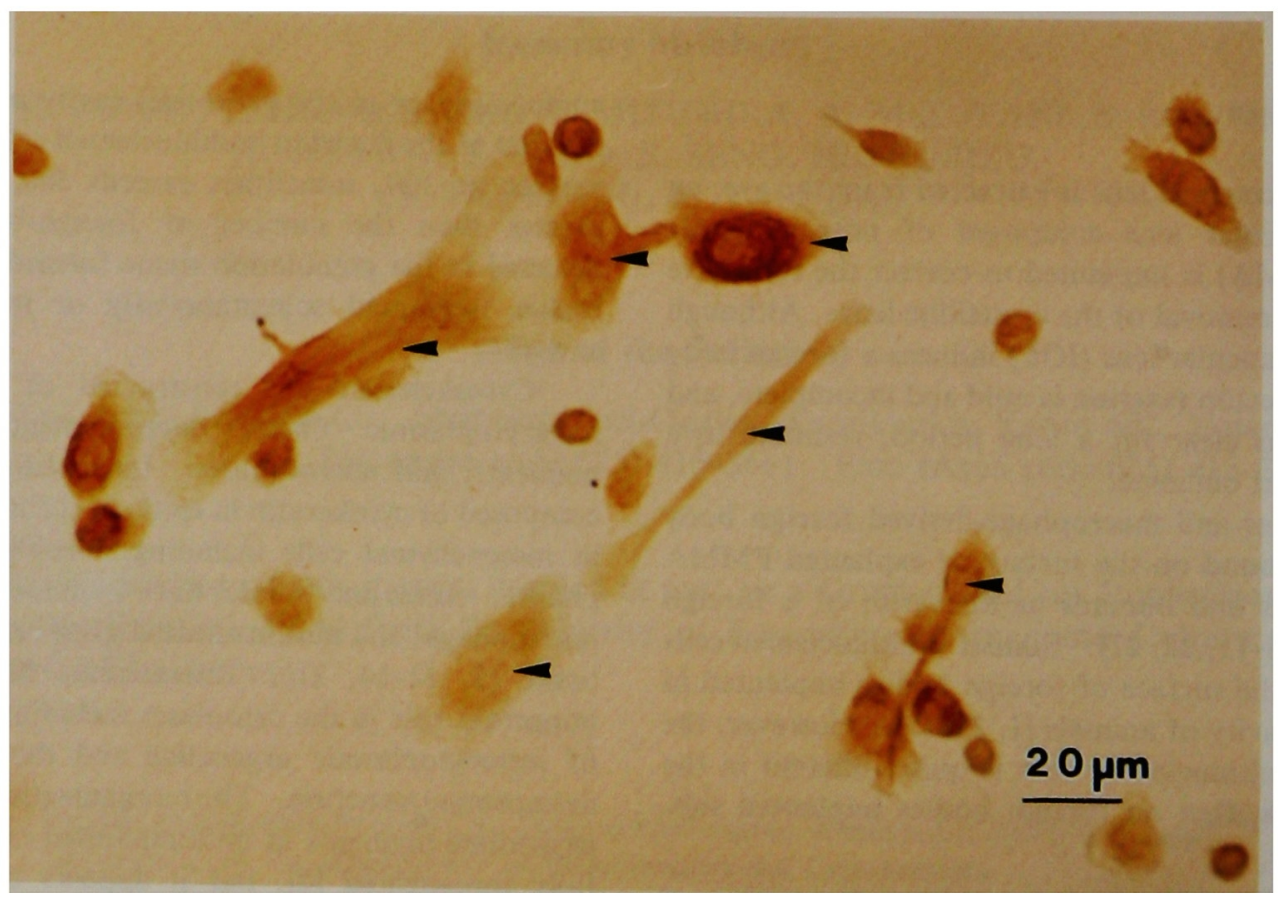

Fig. 1. Macrophages (arrowheads) adhered to the IOL surface. They have vimentin-positive intracytoplasmic fibers. (Indirect Immunostaining, original magnification, $\times 400$. Bar $=20 \mu \mathrm{m}$ ) 


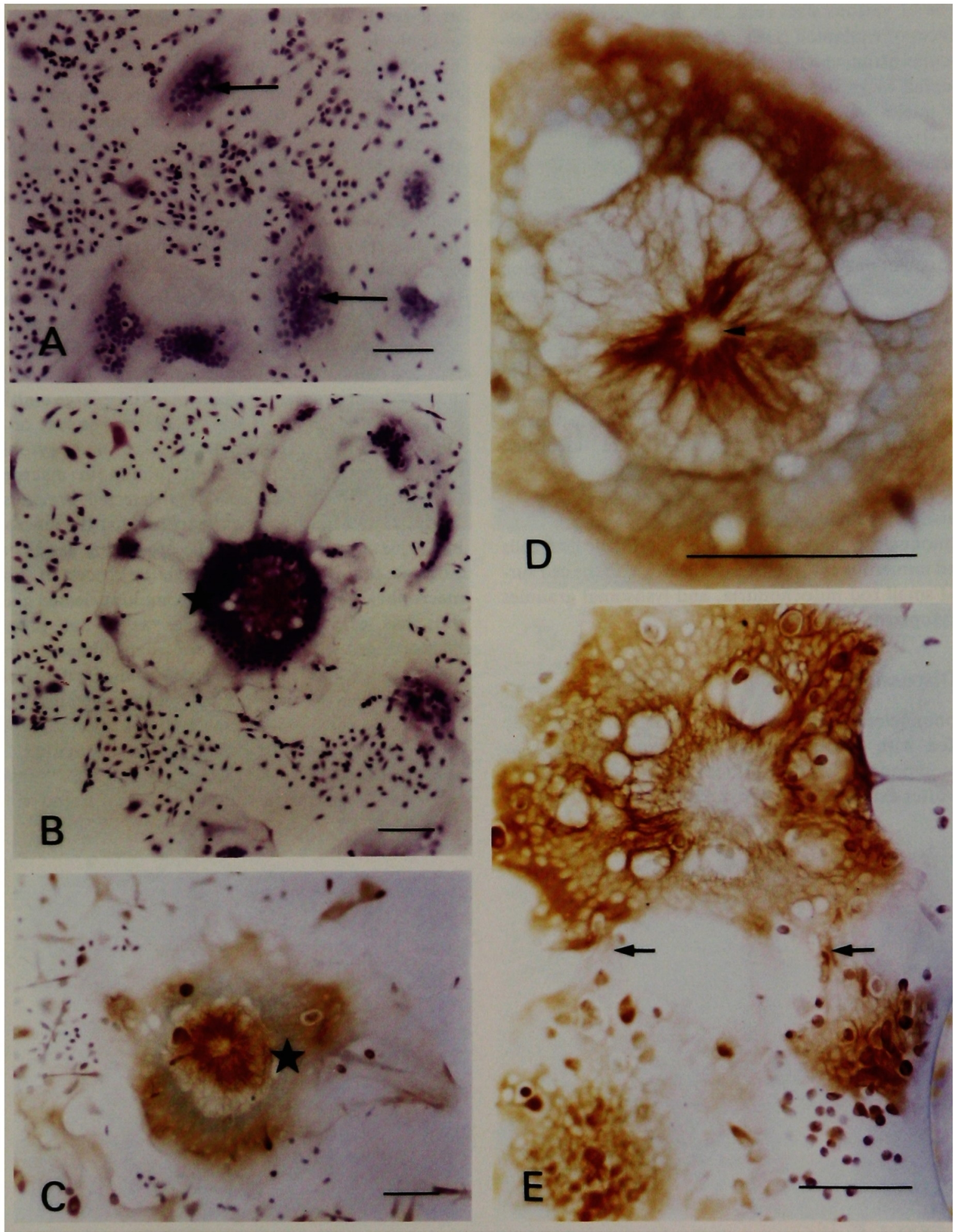

Fig. 2. A \& B: Giant cells observed on the surface of IOLs stained with hematoxylin and eosin. In the small or medium multinucleated cells (arrows), nuclei are visible in the center of the cell; nuclei show a circular distribution in ultralarge multinucleated cells (star), with numerous cytoplasmic vacuoles in the central area of the cell and flattened cytoplasm in the periphery. (Hematoxylin and eosin staining, original magnification, $\times 100$. Bars $=100 \mu \mathrm{m})$ C-E: Immunolocalization of vimentin in cells on the surface of IOLs. C: A foreign body giant cell (star) shows a positive immunoreaction for vimentin. D: At a higher magnification, vimentin intermediate filaments radiated from the center (arrowhead) of the cytoplasm among the cytoplasmic vacuoles, and are densely packed around the cluster of nuclei in a large multinucleated cell. E: Vimentin immunostaining in a large multinucleated cell with circularly arranged nuclei and in a medium giant cell with a cluster of nuclei at the center of the cytoplasm that are probably undergoing fusion (arrows). (Indirect Immunostaining, original magnification, C $\times 100 . \quad$ D $\times 400 . \quad$ E $\times 200 . \quad$ Bars $=100 \mu \mathrm{m})$ 
giant cells of various sizes (Fig. 2A, B) were observed on the surfaces of explanted IOLs. Many of the nuclei showed a circular arrangement, the center of the cytoplasm being occupied by vacuoles in the large multinucleated cells, whereas nuclei were seen in the center of the small and medium-sized multinucleated cells.

Intracytoplasmic fibers positively stained with the anti-vimentin antibody were observed in mononuclear macrophages and multinucleated giant cells (Fig. 2C-E). In macrophages and small multinucleated cells, vimentin intermediate filaments showed a perinuclear accumulation and were elongated toward the periphery of the cytoplasmic processes (not shown). In large multinucleated cells, vimentin fibers radiated from the center of the cytoplasm among the cytoplasmic vacuoles located in the central area of the cell, and were densely packed around the cluster of nuclei. In control staining, no intracytoplasmic fibers immunoreactive for vimentin were identified (Fig. 3).

\section{Transmission electron microscopy}

Mononuclear macrophages and multinucleated cells exhibited numerous cytoplasmic projections on the cell surface and small rod mitochondria, and lysosomal granules in the cytoplasm (Fig. 4A, B).

\section{Discussion}

Mononuclear cells and giant cells are reportedly associated with the deposition of various extracellular matrix components on the surfaces of artificial IOLs implanted after extraction of the crystalline lens, presumably as a result of a foreign body reaction in humans and animals [6-11, 16-20, 22, 27]. Our earlier immunohistochemical study with an anti-macrophage antibody revealed that these mononuclear cells were macrophages, and that the giant cells were of macrophagic origin [10]. A similar cell population composed of macrophages and multinucleated cells has been observed in the granulation tissue formed around coverslips implanted subcutaneously or in the peritoneal cavity of animals $[1,3,13,14,23-25]$. The present study utilizing light and transmission electron microscopy demonstrated that macrophage-derived, large multinucleated cells were formed on the surfaces of PMMA IOLs implanted in rabbit eyes, as previously reported, and that vimentin intermediate filaments were rearranged in the multinucleated cells. The nuclei were arranged in a circular distribution in these large multinucleated cells; the center of the cytoplasm was free of nuclei and occupied by vacuoles. Reorganization of cytoplasmic fibers composed of actin and tubulin in macrophagederived multinucleated cells was detected previously [3, 12-14, 21]. Moudjou et al. reported that the macrophage has a juxtanuclear microtubule-organizing center, a centrosome, that is conserved through the process of fusion of macrophages into a giant cell. A multinucleated giant cell thus has as many centrosomes as it has progenitor macrophages. Although intermediate filaments in such multinucleated cells have not been studied extensively. It has been reported that these filaments have an organizing center $[15,26]$. With respect to the rearrangement of intermediate filaments during the formation of a multinucleated cell $[4,15,26]$, Hiroi and Yamamoto reported the reconstruction of cytokeratin and vimentin

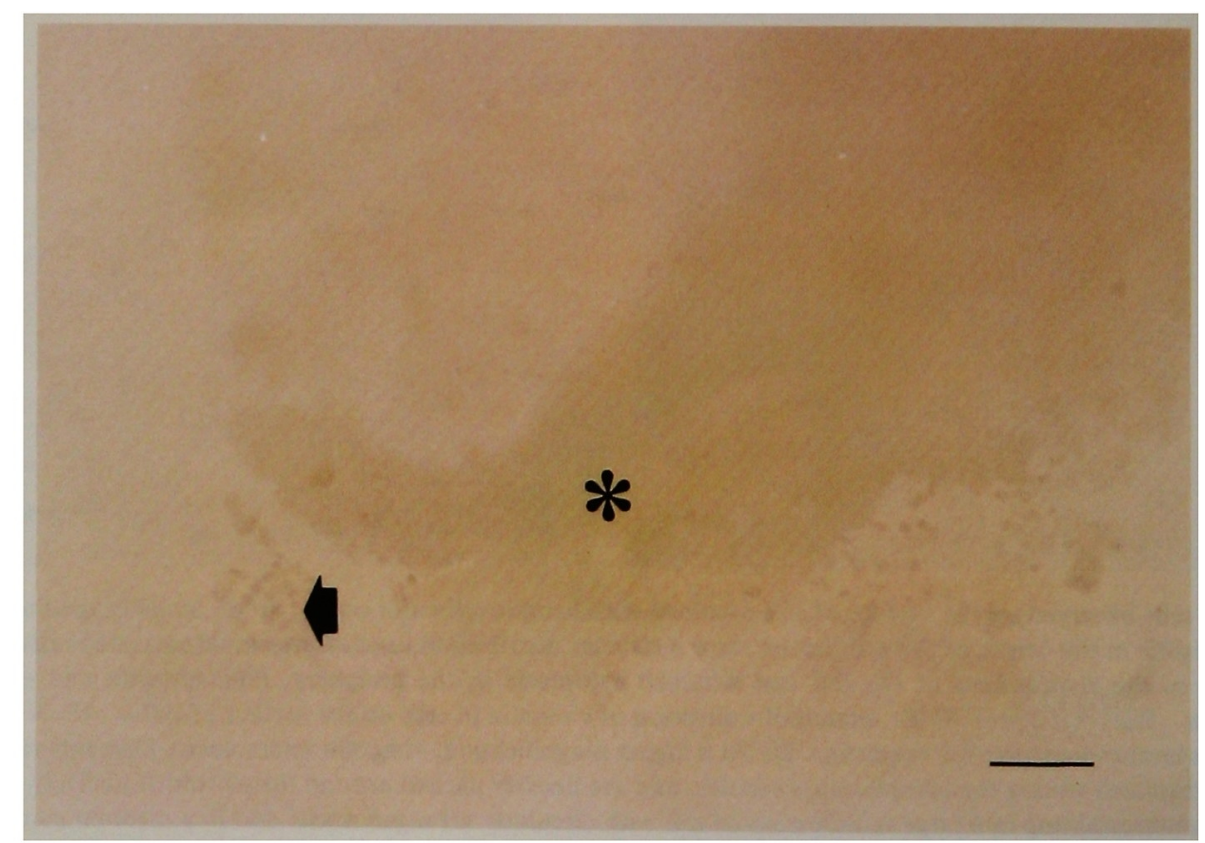

Fig. 3. Control staining with the commision of the primary antibody. Macrophages (arrow) and a giant cell (asterisk) are not stained. (Indirect immunostaining, original magnification, $\times 100 . \quad B a r=100 \mu \mathrm{m}$ ) 


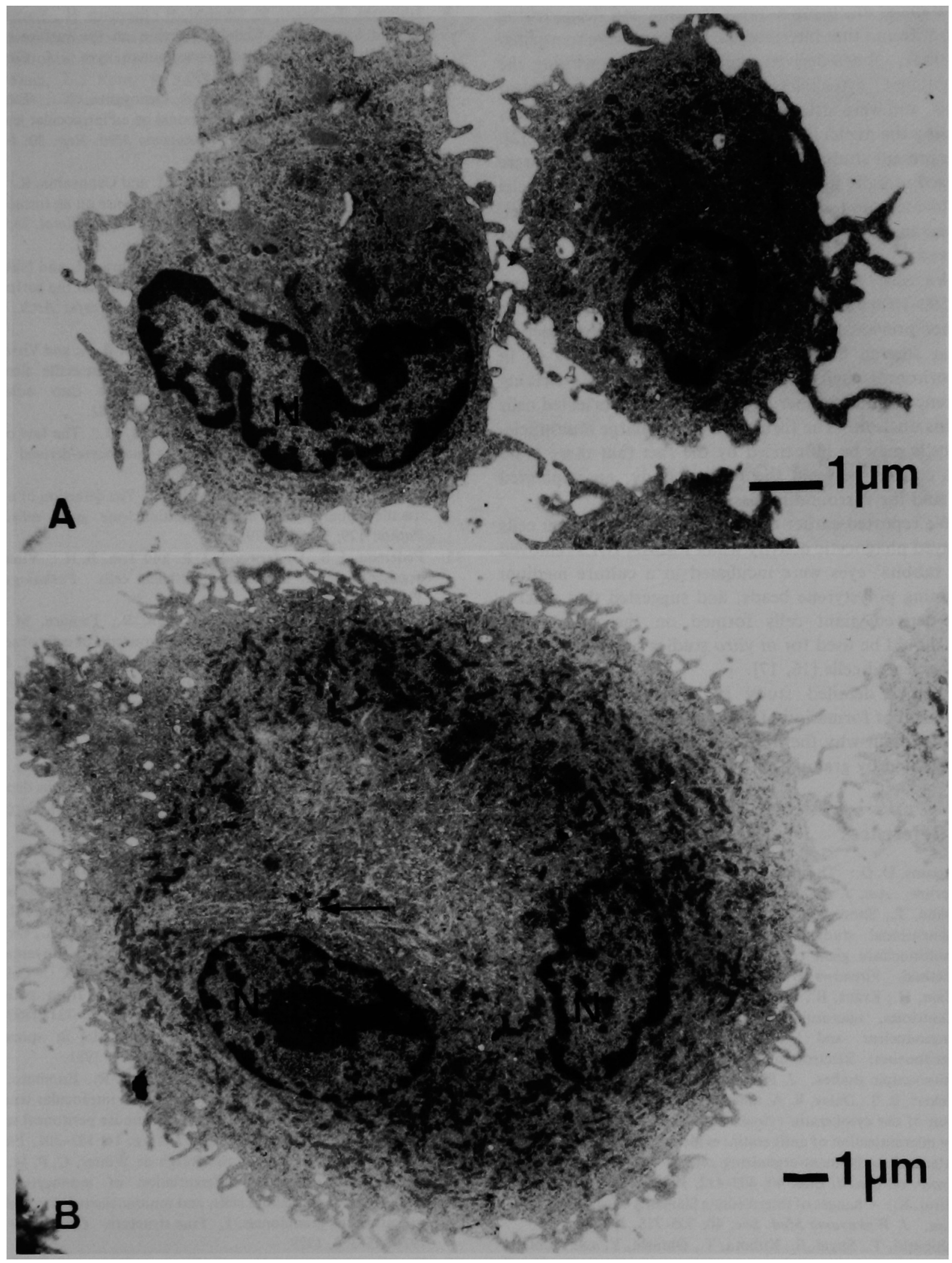

Fig. 4. A \& B: Ultrastructure of macrophages (A) and a binucletead cell (B) detected on the surfaces of IOLs. Small rod mitochondria, lysosomes, and endoplasmic reticulum are visible in the cytoplasm. N: nucleus. Arrow: microtubule-organizing center. (Original magnification, A $\times 12,000$. B $\times 8,000$. Bars $=1 \mu \mathrm{m})$ 
via cell fusion in vitro induced by Herpes simplex virus [5, 28]. Baba et al., using a quick-freezing and deep-etching method, found that intermediate filaments were reorganized in macrophage-derived giant cells that constitute the predominant cytoskeletal components in perinuclear regions, and were attached to intracytoplasmic organellae, including the nuclei in macrophage-derived giant cells [2]. In the present study, vimentin intermediate filaments were arranged radially in the central cytoplasm where no nuclei were located, and were densely packed around the nuclei. No rearranged intermediate filament-organizing center was observed.

We observed large multinucleated cells with more than 500-1000 nuclei. Such ultralarge multinucleated cells had not previously been detected in a rejection reaction against foreign bodies implanted subcutaneously or in the peritoneal cavity. The exact cause of this striking difference in the number of nuclei of multinucleated cells remains unclear. The formation of ultralarge multinucleated cells may be influenced by the fact that there is no direct contact between the foreign body, an implanted $\mathrm{IOL}$, and the surrounding tissues.

We reported earlier that macrophages and giant cells displayed phagocytic activity whan PMMA IOLs removed from rabbits' eyes were incubated in a culture medium containing polystyrene beads, and suggested that macrophage-derived giant cells formed on the surfaces of IOLs should be used for in vitro studies of the biology of multinucleated cells $[16,17]$.

Further detailed study is needed to clarify the mechanism of formation of such large multinucleated cells and to explain why the implanted artificial IOLs are not encapsulated by granulation tissue.

\section{References}

1. Adams, D. O.: The granulomatous inflammatory response. A review. Am. J. Pathol. 84; 164-191, 1976.

2. Baba, T., Shoozawa, N., Hotchi, M. and Ohno, S.: Threedimensional study of cytoskeleton in macrophages and multinucleate giant cells by quick-freezing and deep-etching method. Virchows Archiv B Cell Pathol. 61; 39-47, 1991.

3. Cain, H., Kraus, B., Fringes, M., Osborn, M. and Weber, K.: Centrioles, microtubules and microfilaments in activated mononuclear and multinuclear macrophages from rat peritoneum: Electron-microscopic and immunofluorescence microscopic studies. J. Pathol. 133; 301-323, 1981.

4. Eckert, B. S., Daley, R. A. and Parysek, L. M.: In vivo disruption of the cytokeratin cytoskeleton in cultured epithelial cells by microinjection of antikeratin: evidence for the presence of an intermediate-filament-organizing center. Cold Spring Harbor Symp. on Quant. Biol. 40; 403-412, 1982.

5. Hiroi, K.: Changes of intermediate filaments during virus infection. J. Wakayama Med. Soc. 40: 705-716, 1989.

6. Ishibashi, T., Sugai, S., Kubota, T., Ohnishi, Y. and Inomata, H.: Cytopathology of early cellular response on implanted lenses in monkeys. Graefes Arch. Clin. Exp. Ophthalmol. 227; 470-475, 1989.

7. Ishibashi, T., Sugai, S., Kubota, T., Ohnishi, Y. and Inomata H.: Cellular adhesiveness on implanted lenses in monkeys.
Graefes Arch. Clin. Exp. Ophthalmol. 228; 356-362, 1990.

8. Ishibashi, T., Sugai, S., Ohnishi, Y., Inomata, H., Yoshitomi, F. and Sasaki, K.: Cellular reaction on the surface of intraocular lenses removed from human eyes. $J$. Cataract Refract. Surg. 16; 751-754, 1990.

9. Kanagawa, R., Ohmi, S. and Uenoyama, K.: Electron microscopic study of cellular response on an intraocular lens implanted in the rabbit eye. Wakayama Med. Rep. 30; 61-65, 1988.

10. Kanagawa, R., Ohmi, S., Tamura, M. and Uenoyama, K.: Immunohistochemical study of cellular response on an intraocular lens implanted in the rabbit eye. Jpn. J. Ophthalmol. 33; 337342, 1989.

11. Kanagawa, R., Saika, S., Ohmi, S., Tamura, M. and Nakano, T.: Presence and distribution of fibronectin on the surface of implanted intraocular lenses in rabbits. Graefes Arch. Clin. Exp. Ophthalmol. 228; 398-400, 1990.

12. Lehto, V. P., Hovi, T., Vartio, T., Badley, R. A. and Virtanen, I.: Reorganization of cytoskeletal and contractile elements during transition of human monocytes into adherent macrophages. Lab. Invest. 47; 391-399, 1982.

13. Moudjou, M., Lanotte, $M$. and Bornens, $M$. .: The fate of the centrosome-microtubule network in monocyte-derived giant cells. J. Cell Sci. 94; 237-244, 1989.

14. Papadimitriou, J. M. and Rigby, P. J.: The detection of a contractile apparatus in murine multinucleate giant cells. $J$. Pathol. 129; 91-97, 1979.

15. Pederson, J. S., Underwood, J. R. and Toh, B. H.: Vimentin organizing center in cultured epithelial cells. Pathology 16; 393-395, 1984.

16. Saika, S., Uenoyama, S., Kanagawa, R., Tamura, M. and Uenoyama, K.: Phagocytosis and fibronectin of cells observed on intraocular lenses. Jpn. J. Ophthalmol. 36; 184-191, 1992.

17. Saika, S., Tamura, M., Nakao, T., Kanagawa, R., Uenoyama, K. and Hiraoka, J.: Experimental study of phagocytic activity of cells observed on implanted intraocular lenses. Eur. $J$. Implant Refract. Surg. 3; 87-93, 1991.

18. Saika, S., Tamura, M., Uenoyama, K., Yamanaka, A., Ohkubo, K., Fukuda, K. and Iwane, H.: Collagenous deposits on explanted intraocular lenses. J. Cataract Refract. Surg. 18; 195-199, 1992.

19. Saika, S., Tonoe, O., Kanagawa, R., Uenoyama, K., Yamanaka, A., Fukuda, K. and Iwane, H.: Immunohistochemical study of deposits on intraocular lenses explanted from human eyes. Jpn. J. Ophthalmol. 35; 96-101, 1992.

20. Saika, S., Kobata, S., Yamanaka, O., Yamanaka, A., Okubo, K., Oka, T., Hosomi, M., Kano, Y., Ohmi, S., Uenoyama, S., Tomura, M., Kanagawa R. and Uenoyama, K.: Cellular fibronectin on intraocular lenses explanted from patients. Graefes Arch. Clin. Exp. Ophthalmol. 231; 718-721, 1993.

21. Trotter, J.A.: The organization of actin in spreading macrophages. Exp. Cell Res. 132; 235-248, 1981.

22. Uenoyama, K., Matoba, M., Kanagawa, R., Enomoto, Y., Tamura, M. and Ohmi, S.: Experimental intraocular lens implantation in the rabbit eye and in the mouse peritoneal space. I, II, and III. J. Cataract Refract. Surg. 14; 187-201, 1988.

23. Van der Rhee, H. J., Van der Burgh-de Winter, C. P. M. and Daems, W. Th.: The differentiation of monocytes into macrophages, epithelioid cells, and multinucleated giant cells in subcutaneous granulomas. I. Fine structure. Cell Tissue Res. 197; 355-378, 1979.

24. Van der Rhee, H. J., Van der Burgh-de Winter, C. P. M. and Daems, W. Th.: The differentiation of monocytes into macrophages, epithelioid cells, and multinucleated giant cells in subcutaneous granulomas. II. Peroxidatic activity. Cell Tissue Res. 197; 379-396, 1979. 
25. Van der Rhee, H. J., Van der Burgh-de Winter, C.P. M., Tijissen, J. G. P. and Daems, W. Th.: Comparative study on peroxidatic activity in inflammatory cells on cutaneous and peritoneal implants. Cell Tissue Res. 197; 397-412, 1979.

26. Wang, E., Roos, D. S., Heggeness, M. H. and Choppin, P. W.: Function of cytoplasmic fibers in syncytia. Cold
Spring Harbor Symp. on Quant. Biol. 39; 997-1012, 1981.

27. Wolter, J.R.: Cytopathology of intraocular lens implantation. Opathalmology 92; 135-142, 1985.

28. Yamamoto, Y.: Alteration and function of intermediate filaments with syncytial cells induced by herpes simplex virus infection. J. Wakayama Med: Soc. 44; 105-112, 1993. 